\title{
Augmented Vessels for Pre-operative Preparation in Endovascular Treatments
}

\author{
Wilbur C.K. Wong ${ }^{1}$, Albert C.S. Chung ${ }^{1}$, and Simon C.H. Yu ${ }^{2}$ \\ 1 Department of Computer Science, \\ The Hong Kong University of Science and Technology, \\ Clear Water Bay, Kowloon, HK \\ \{cswilbur, achung\}@cs.ust.hk \\ 2 Department of Diagnostic Radiology and Organ Imaging, \\ Prince of Wales Hospital, Shatin, NT, HK \\ simonyu@cuhk. edu.hk
}

\begin{abstract}
Three-dimensional rotational angiography is a very useful tool for accessing abnormal vascular structures related to a variety of vascular diseases. Quantitative study of the abnormalities could aid the radiologists to choose the appropriate apparatuses and endovascular treatments. Given a segmentation of an angiography, effective quantitation of the abnormalities is attainable if the abnormalities are detached from the normal vasculature. To achieve this, a novel method is presented, which allows the users to construct imaginary disease-free vessel lumens, namely augmented vessels, and demarcate the abnormalities on the fly interactively. The method has been tested on several synthetic images and clinical datasets. The experimental results have shown that it is capable of separating a variety of abnormalities, e.g., stenosis, saccular and fusiform aneurysms, from the normality.
\end{abstract}

\section{Introduction}

An endovascular treatment is a therapy performed inside vascular structures with the assistance of 2D angiography and micro-catheter. During the treatment, there is a few imaging technologies that can provide 3D vascular morphology. Three-dimensional rotational angiography (3D RA) is one of these technologies, which is capable of producing high resolution lumen images in isotropic voxel\$1. As such, 3D RA is a very useful tool for accessing abnormal vascular structures of a variety of pathologies. Given a vascular segmentation of a 3D RA image, it would be more effective to perform quantitation on the abnormalities if they are detached from the normal vasculature. This makes further processing exclusively on the abnormal structures possible. Therefore, this could allow interactive or automatic quantitative analysis of the abnormalities, and aid the radiologists to choose the appropriate apparatuses and treatments to cure the vascular diseases.

To the best of our knowledges, there is relatively little literature on separating abnormal vascular structures from the normal lumen in angiographies.

\footnotetext{
${ }^{1}$ We acquire the 3D RA images with a Philips Integris imager.
} 
Several researchers have suggested to detach a saccular aneurysm by defining the aneurysmal neck [1], [2] or by identifying the mesh covering the saccular aneurysm from the surface mesh of the vasculature [3. Their algorithms, however, are restricted to a particular type of abnormality - saccular aneurysm.

In this paper, we present a novel approach to detaching a variety of vascular abnormalities, viz. stenosis, saccular and fusiform aneurysms, from the normal vasculature. We take a very different approach as compared with the published methodologies. Instead of manipulating the abnormal structures with sophisticated algorithms, we detach the abnormalities by explicitly modeling their opposite - normal vessels. Our method provides interactive tracking of imaginary disease-free vessel lumens, namely augmented vessels, and the demarcation of the abnormalities is performed on the fly. The method has been validated with synthetic images by comparing the results to a manual delineation of the abnormalities and tested on several clinical datasets.

\section{Methods}

In order to track the augmented vessels and detach the abnormalities from the normal vasculature, we have to preform the following tasks: (a) segment the vascular structures, (b) estimate the lumen diameters, (c) extract the centerline of the lumen, (d) track the augmented vessels and (e) detach the abnormalities.

\subsection{Vascular Segmentation}

In the image acquisition of $3 \mathrm{D}$ RA, the radiologist injects contrast agents in the vessel to fill the pathology of interest so as to image the vessel lumen in the angiography. Because of the application of the contrast agents, the blood vessel lumen is stood out on the background. This makes the segmentation of the lumen a less difficult task to accomplish. According to our experiments, a satisfactory 3D RA vascular segmentation can be produced by a global thresholding after the noisy angiography is smoothed with an edge-preserving filter. In this work, we applied the trilateral filter [4] to the angiography for denoising prior to the segmentation.

\subsection{Lumen Diameter Estimation}

In order to model the blood vessel lumen with a circular cross-sectional tub 2 , we have to estimate the lumen diameter. We employ Saito and Toriwaki's Euclidean distance transformation algorithm [6] to calculate the Euclidean distance (ED) from each voxel inside the lumen to the nearest boundary of the lumen (aka ED map). Intuitively, the distance value approximates the radius of the largest sphere that can be completely enclosed within the lumen at the corresponding voxel. Therefore, the diameters of the vessel lumen can be estimated from the ED map and equal twice the EDs at the voxels along the vessel centerline.

\footnotetext{
${ }^{2}$ The assumption - blood vessel is a circular cross-sectional tubular object - is a commonly used hypothesis in medical image processing community [5].
} 


\subsection{Centerline Extraction}

We use Palágyi et al. sequential 3D thinning algorithm [7] to extract voxels along the centerline of the blood vessel lumen. Their thinning algorithm iteratively reduces an object to produce an approximation to the object skeleton. The algorithm is simple and easy to implement, and, more importantly, the topology of the object is preserved in the skeleton. However, it may take several iterations to produce the skeleton if the object is large. Unfortunately, it is the case for the 3D RA images in a clinical environment - typically, the object of interest occupies 170,000 voxels in a $256 \times 256 \times 256$ voxels volume with a $30 \mathrm{~mm}$ field of view in each dimension.

Providentially, in this work, we are not aiming at extracting the complete skeleton of the vessel. Indeed, our objective is to provide a few $3 \mathrm{D}$ points that are located at the centerline of the blood vessel to the user for the initialization of the tracking of the augmented vessel. As such, we can resample the binary image volume with a larger voxel size to reduce the computation of the thinning algorithm. In this work, a voxel size of $1 \times 1 \times 1 \mathrm{~mm}^{3}$ is used. This is because the diameter of the vessel of interest usually ranges from $2 \mathrm{~mm}$ to $10 \mathrm{~mm}$. Therefore, in the resampled image volume, the diameter of the lumen is about $2-10$ voxels, and the object of interest occupies only 270 voxels. In general, the algorithm takes less than 1 sec. to extract the skeleton on a $2.6 \mathrm{GHz} \mathrm{PC}$.

Once the skeleton points in the low resolution image volume are extracted, we can search for the 3D points that are located at the centerline of the lumen within a $3 \mathrm{D}$ spatial window of $1 \mathrm{~mm}$ in each dimension 3 . The centerline points are characterized by the local maxima in the ED map of the original binary image volume. In order to achieve subvoxel accuracy in the search of these points, we perform tricubic interpolation on the ED map and seek the position with the maximum ED within the spatial window at each skeleton point in a sampling space $1 / 8$ of the original voxel size.

\subsection{Interactive Tracking of Augmented Vessel}

After the centerline points are extracted, we present them together with the $3 \mathrm{D}$ surface mesh of the vessel lumen to the usen. Then the user has to select three centerline points at each end of the vessel of interest. The vessel of interest usually connects directly to the part of pathology of interest, such as an aneurysm or a stenosis, between the two vessel ends. The objective of the tracking task is to follow the trajectory of the augmented vessel. By tracking this imaginary trajectory, we can distinguish the abnormalities from the normal vasculature.

Instead of the conventional B-spline representation [2], [8], a cardinal splin 5 (with zero tension) is employed to approximate the vessel trajectory, because we desire the control points to be laid on the spline/vessel itself. This representation

\footnotetext{
${ }^{3}$ We assume that the skeleton points extracted are close to the vessel centerline.

4 The Marching Cube algorithm is used to compose the surface mesh for visualization.

${ }^{5}$ Cardinal spline is a special type of the well-known TCB-spline (aka Kochanek-Bartels spline) with local tension control only [9].
} 
is more intuitive in both the computation of the spline deformation and the presentation to the user.

The six points selected by the user are fixed throughout the deformation. They avoid high curvature at the ends of the deformed spline and allow a robust estimation of the lumen diameter 6 . The lumen diameter at one end can be approximated from the ED map and equals twice the average of the EDs at the corresponding three points. For the lumen diameters in between the two ends, linear interpolation is performed.

In addition to these fixed points, the user has to specify the number of movable points inserted in between the two ends of the spline. These non-fixed points characterize the degree of freedom of the trajectory approximation. In general, two to four movable points are sufficient for the approximation, since the desired imaginary trajectory is usually simple in practice.

Concerning the spline deformation, we apply Kass et al. active contour models (aka snakes) [10] with the dynamic programming implementation proposed in [11. In this work, the energy functional to be minimized is defined as,

$$
E_{\text {snake }}^{*}=\int_{0}^{1}\left(\beta\left|v_{s s}(s)\right|^{2}+E_{\text {image }}(v(s))\right) d s,
$$

where $s$ is the parametric variable, $v(\cdot)$ denotes the cardinal spline, $\beta$ defines the stiffness of the spline (equals 10 in this work) and $E_{\text {image }}(\cdot)$ defines the image forces. The term $E_{\text {image }}$ plays an important role in driving the spline to follow the trajectory of the augmented vessel. It encourages the imaginary vessel to be confined within the imaged lumen. The image forces are expressed as,

$$
E_{\text {image }}(v(s))=\iiint_{G(v(s))}(1-f(x, y, z)) d x d y d z
$$

where $G(v(s))$ denotes a sphere located at position $v(s)$ with its diameter equal to the lumen diameter approximated at $v(s)$. The function $f(\cdot)$ denotes the binary image volume, $f(x, y, z)=1$ if the point $(x, y, z)$ is laid inside the vascular structure; otherwise, $f(x, y, z)=0$.

Figure 1(a) shows a synthetic object. Figure 1(b) shows the object in semitransparent with a few points on the centerline of the object structures extracted as described in Section 2.3 The user-selected six fixed points are in darker color as highlighted by the arrows. Figure 1(c) shows the imaginary lumen before the spline deformation, whereas Figure $\mathbb{1}(\mathrm{d})$ shows the lumen after the deformation. It is indicated that, with the proposed energy functional (see Equations 1 1and 2), the cardinal spline (i.e. centerline of the imaginary lumen) deforms and follows the trajectory of the object structure. Moreover, the imaginary lumen is within the confines of the object (even in the high curvature region).

\footnotetext{
${ }^{6}$ It is assumed that the three points at each end are close to each other and are located at the centerline of the normal blood vessel lumen.
} 


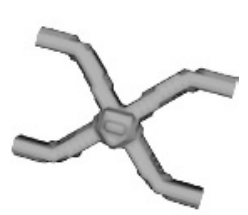

(a)

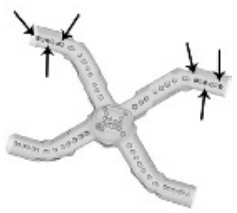

(b)

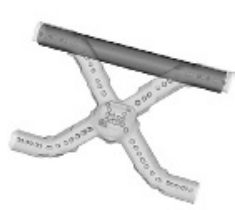

(c)

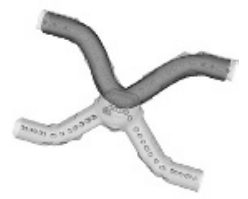

(d)

Fig. 1. Interactive tracking of a synthetic lumen. (a) 3D surface model of the object; (b) object in semi-transparent with a few centerline points presented, the user-selected six fixed points are in darker color as highlighted by the arrows; (c) imaginary lumen before the spline deformation; and (d) imaginary lumen after the deformation.

\subsection{Formation of Augmented Vessel and Separation of Abnormalities}

Once the imaginary lumen is tracked, the voxels that contribute to the abnormalities can be distinguished from the (imaginary) normal vascular voxels. The abnormal voxels can be picked up with a user-defined seed by applying a connectivity filter. As a consequence, the augmented vessel is obtained. In the case of an aneurysm, the abnormalities are the surplus vascular voxels that are attached to the augmented vessel. Whereas for the case of a stenosis, the abnormalities are the voxels that are absent from the augmented vessel.

\section{Evaluation}

The method is applied to three synthetic images for evaluation. We have also compared the accuracy of our methodology to a manual delineation of the abnormalities on one of the synthetic images. The design of the synthetic images takes several typical pathologies into consideration: cerebral aneurysm, abdominal aortic aneurysm and arterial stenosis. They are created to emulate the field of view of the region of interest $(30-100 \mathrm{~mm})$ and the resolution $(256 \times 256 \times 256$ voxels) of typical 3D RA images.

Figure 2(a) shows the 3D surface model of the synthetic saccular cerebral aneurysm. Figure 2(b) shows the abnormalities (highlighted by the arrow) detached from the normal vascular structures with our method. Figure 2(c) shows the two imaginary vessel lumens (highlighted by the arrows) on top of the augmented vessel. While Figures 2(d)-(f) and Figures 2(g)-(i) show similar illustrations for the synthetic abdominal aortic aneurysm and arterial stenosis, respectively. It is evident that by constructing the imaginary normal lumen(s), we can separate the abnormalities from the normality with ease.

In a further study of the method, we have compared the accuracy of the abnormality estimation with our method to a manual delineation on the synthetic cerebral aneurysm image. The manual delineation is performed assuming the normal vessel lumen is a T-shape structure. To demonstrate the robustness of our method in handling object oriented at different angles, we have built the 


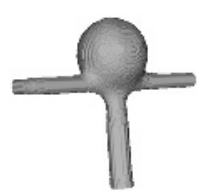

(a)

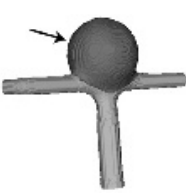

(b)

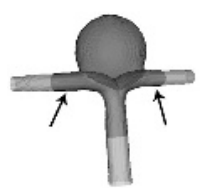

(c)

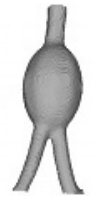

(d)

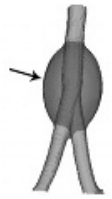

(e)

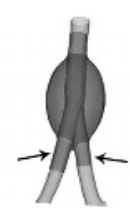

(f)

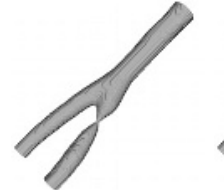

(g)

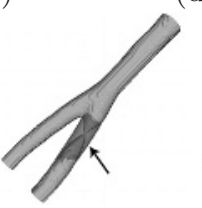

(h)

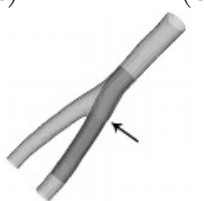

(i)

Fig. 2. Synthetic images of different typical pathologies: (a)-(c) cerebral aneurysm, (d)-(f) abdominal aortic aneurysm and (g)-(i) arterial stenosis. (a, d, g) 3D surface model of the pathology; (b, e, h) abnormalities, highlighted by the arrow, detached from the normal vasculature with our method; and (c, f, i) imaginary vessel lumen(s), highlighted by the arrow(s), on top of the augmented vessel.

augmented vessels in the image volumes with the synthetic cerebral aneurysm oriented at different angles $\left(0^{\circ}-90^{\circ}\right.$ with a step size of $\left.15^{\circ}\right)$ around the axis perpendicular to the T-shape structure. It is found that the volumetric estimation of the synthetic aneurysm produced by our method (sensitivity: $99.32 \%$ and specificity: $99.42 \%$ ) is, in general, more accurate than the manual delineation (sensitivity: $94.63 \%$ and specificity: $99.99 \%$ ).

\section{Results}

We have applied our method to three 3D RA datasets. These datasets were acquired by a Philips Integris imager at the Department of Diagnostic Radiology and Organ Imaging, Prince of Wales Hospital, Hong Kong. The size of the image volume is $256 \times 256 \times 256$ voxels with the field of view ranges from $30-50 \mathrm{~mm}$. The pathology of interest in the datasets is cerebral aneurysm with neck ranges from narrow $(\leq 4 \mathrm{~mm})$ to wide $(>4 \mathrm{~mm})$ as classified in 12 .

The first two columns of the images presented in Figure 3 show the results obtained by our method. The first column images show the vascular structures with the abnormalities in semi-transparent, highlighted by the arrows. While the second column images present the imaginary lumens, highlighted by the arrows, on top of the augmented vessels. The third and the forth column images show the scans before and after the embolizations, respectively. The Guglielmi detachable coils (GDC) are displayed as the brighter objects in the post-operative scans.

It is indicated that the GDC embolized volumes correspond to the abnormalities detached by our method in the pre-operative scans. One may find that there is an aneurysm left out in the embolization as observed in Figure 3(h), highlighted by the arrow. This is because the aneurysm is too small $\left(2.5 \mathrm{~mm}^{3}\right)$ to be treated in the endovascular treatment, according to the radiologist-in-charge. 


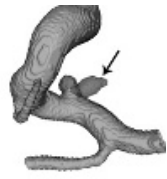

(a)

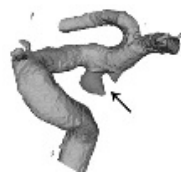

(e)

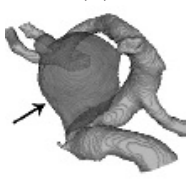

(i)

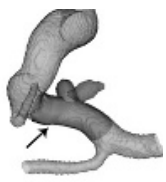

(b)

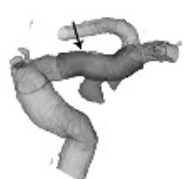

(f)

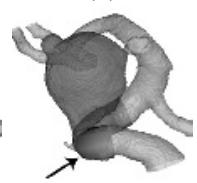

(j)

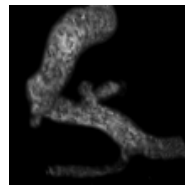

(c)

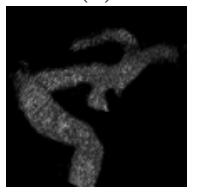

(g)

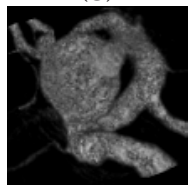

(k)

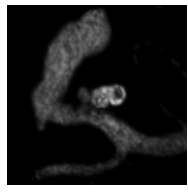

(d)

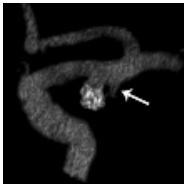

(h)

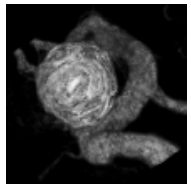

(m)

Fig. 3. Clinical datasets with cerebral aneurysms. Column 1 (a, e, i): vascular structures with the abnormalities in semi-transparent, highlighted by the arrows; Column 2 (b, f, j): imaginary lumens, highlighted by the arrows, on top of the augmented vessels; Column 3 (c, g, k): pre-operative scans; and Column 4 (d, h, m): post-operative scans.

Finally, for all the experiments conducted in this paper, the purposed method requires less than 15 sec. computation time 7 and only a few mouse clicks to build the augmented vessels and detach the abnormalities. It is also worth mentioning that our method is not restricted to separate abnormalities of pathology related to saccular aneurysm. We believe that our interactive tools can be applicable to other pathologies, for instance, fusiform aneurysm and stenosis, as illustrated with the synthetic images presented in Section 3 .

\section{Conclusions}

A novel method that can allow users to separate a variety of vascular abnormalities from the normal vasculature is presented. By detaching the abnormalities, exclusive post-processing on the abnormal vascular structures becomes feasible. This allows effective quantitation of the structures and could aid the radiologists to choose the appropriate apparatuses and endovascular treatments to cure the vascular diseases that cause the abnormalities. Our method starts with vascular segmentation of 3D RA images, followed by lumen diameter estimation and centerline extraction. A few points on the lumen centerline and the surface mesh of the vasculature are then displayed to the user. The six user-selected fixed points at each end of the vessel of interest and the few inserted movable points

\footnotetext{
7 The computation time does not include the time spent on filtering and segmentation, since they are not the foci of this work.
} 
define a cardinal spline which deforms to follow the trajectory of the imaginary disease-free vessel lumen, namely augmented vessel. Once the augmented vessel is tracked, the abnormalities can be separated on the fly interactively.

The proposed method has been tested on several synthetic images and clinical datasets. It is validated with visual comparisons instead of other non-visual means. This is because in the clinical 3D RA datasets manual delineation of the abnormal structures from the vascular segmentations is not only difficult, if not impossible, but also inaccurate. Especially when the aneurysmal necks are out-of-plane with respect to the image slices, not to mention if the necks are wide and cannot be defined on planes. The experimental results have shown that the novel method is applicable for separating abnormalities of different kinds of pathologies, viz. stenosis, saccular and fusiform aneurysms.

Several analyses of the proposed method are of the current research interests. Experiments on clinical datasets that contains stenoses and abnormalities in much complex shape, for instance, saccular aneurysm that grows at the inner angle of a high curvature vessel lumen, are being conducted. Studies of the volumetric measurements of the detached abnormalities are being carried out.

\section{References}

1. van der Weide, R., Zuiderveld, K.J., Mali, W.P.T.M., Viergever, M.A.: CTAbased angle selection for diagnostic and interventional angiography of saccular intracranial aneurysms. IEEE TMI 17 (1998) 831-841

2. Wilson, D.L., Royston, D.D., Noble, J.A., Byrne, J.V.: Determining X-ray projections for coil treatments of intracranial aneurysms. IEEE TMI 18 (1999) 973-980

3. McLaughlin, R.A., Noble, J.A.: Demarcation of aneurysms using the seed and cull algorithm. In: MICCAI. Volume 2488 of LNCS., Springer-Verlag (2002) 419-426

4. Wong, W.C.K., Chung, A.C.S., Yu, S.C.H.: Trilateral filtering for biomedical images. In: IEEE ISBI. (2004) 820-823

5. Aylward, S., Bullitt, E.: Initialization, noise, singularities, and scale in height ridge traversal for tubular object centerline extraction. IEEE TMI 21 (2002) 61-75

6. Saito, T., Toriwaki, J.I.: New algorithms for Euclidean distance transformations of an n-dimensional digitized picture with applications. PR 27 (1994) 1551-1565

7. Palágyi, K., Sorantin, E., Balogh, E., Kuba, A., Halmai, C., Erdöhelyi, B., Hausegger, K.: A sequential 3D thinning algorithm and its medical applications. In: IPMI. Volume 2082 of LNCS., Springer-Verlag (2001) 409-415

8. Frangi, A.F., Niessen, W.J., Hoogeveen, R.M., Walsum, T.V., Viergever, M.A.: Model-based quantitation of 3-D magnetic resonance angiographic images. IEEE TMI 18 (1999) 946-956

9. Kochanek, D., Bartels, R.: Interpolating splines with local tension, continuity and bias control. Comput. Graph. 18 (1984) 33-41

10. Kass, M., Witkin, A., Terzopoulos, D.: Snakes: Active contour models. IJCV 1 (1987) 321-331

11. Amini, A.A., Weymouth, T.E., Jain, R.C.: Using dynamic programming for solving variational problems in vision. IEEE PAMI 12 (1990) 855-867

12. Zubillaga, A.F., Guglielmi, G., Vinuela, F., Duckwiler, G.R.: Endovascular occlusion of intracranial aneurysms with electrically detachable coils: Correlation of aneurysm neck size and treatment results. AJNR 15 (1994) 815-820 